\title{
Multi-functional Smart Skin for Multi-dimensional Perception for Humanoid Robots
}

\author{
Yanning Dai ${ }^{1}$ and Shuo Gao ${ }^{1,2, *}$ \\ ${ }^{1}$ School of Instrumentation Science and Optoelectronic Engineering, Beihang University, Beijing, 100083, China \\ ${ }^{2}$ Beijing Advanced Innovation Center for Big Data-Based Precision Medicine, Beihang University, Beijing, 100083, \\ China \\ *shuo_gao@buaa.edu.cn
}

\begin{abstract}
Artificial smart skins capable of interacting with people and sensing environmental stimuli have become a research topic in humanoid robotic applications. However, previously reported architectures suffer difficulties in achieving multi-dimensional sensing in a simple structure with a low system cost. To address this issue, in this paper, an artificial smart skin constructed with polyimide/copper/polyvinylidene fluoride (PVDF) is presented for detecting 2D-position, proximity, dynamic force, and humidity via a smart combination of piezoelectric- and capacitive-effects. The proposed system achieves overall force and capacitive sensitivities of $0.051 \mathrm{~N}$ and $8.7 \mathrm{fF}$; the humidity measurements show a responsivity at $0.20 \% / \mathrm{RH} \%$ over a relative humidity range of $10 \%-90 \% \mathrm{RH}$. And a follow-up filtering algorithm is proposed to separate the stimuli associated with capacitance changes (position, proximity, and humidity). This simple-structured device supports multiple functions with its low system cost, thus advancing the field of robotics smart skins.
\end{abstract}

Keywords-artificial smart skin; position and hover touch recognition; force detection and humidity sensing

\section{INTRODUCTION}

With the rapid development of electronics and computer science, humanoid robots have been engaging in our daily lives since the early 21 st century [1-6]. To enable humanoid robots to provide advanced services, using human skininspired artificial smart skin for robots to help them interact with users and perceive environmental stimuli has received increased attention in recent years [7-10]. Variety techniques have been proposed and demonstrated in the literature to enable robots to sense multi-dimensional physical changes, such as pressure [7-8] and temperature [9]. However, most of the reported works focus on single-dimensional sensing, such as force sensing, which could be picked up by the human skin and delivered to the brain for further processing. To address this, layers of different functionalities are integrated for multidimensional sensing, e.g., force, and temperature sensing layers are stacked in [9]. However, this process increases front-end and circuitry complexity, as well as system power consumption and component costs (especially when a large area is required), thus limiting its successfully extensive use in commercial products. Hence, highly desired attributes for

Research funds from the National Natural Science Foundation (grant number 61803017, 61827802), the "Double FirstClass" university project, and Beihang University Excellent 100 project.

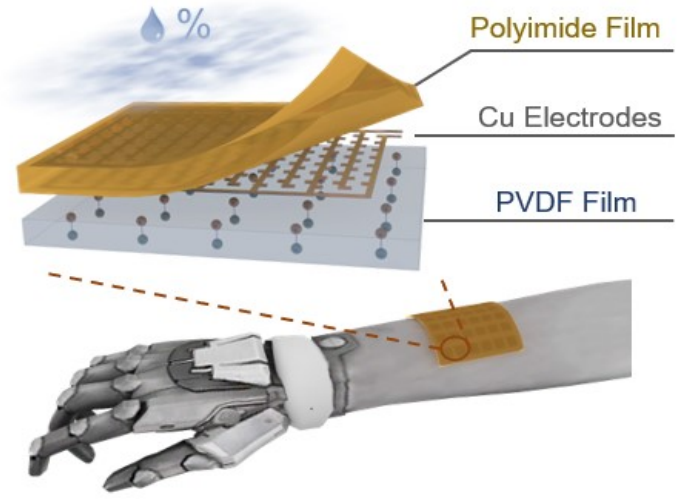

Fig. 1. A conceptual depiction of the proposed multifunctional smart skin.

smart skins in the foreseeable future should be multifunctional, simple structured, and low cost.

To demonstrate this possible development trend, in this article, we exhibit a polyimide/copper/polyvinylidene fluoride (PVDF) structured smart skin for multidimensional sensing (including position, proximity, force and humidity), which is enabled by smart utilization of dielectric property of polyimide layer and piezoelectric of the PVDF layer. This simple structured multifunctional layer only consists of commercialized materials and does not require cleanroom fabrication conditions, potentially allowing massive production by assembly lines. Below we will explain how this smart skin advances the related research area, its working principles, and the corresponding results.

\section{METHODOLOGY AND SYSTEM}

\section{A. Device Architecture}

The developed device is composed of three layers (polyimide/copper/PVDF), as illustrated in in Fig. 1. The top polyimide layer, with multi-hole structures, serves as the humidity sensing material, of which the dielectric permittivity will increase under moisture absorption. Interdigital copper electrodes are fabricated on the polyimide film to form mutual capacitors in a single layer. Touch events and hover distances 


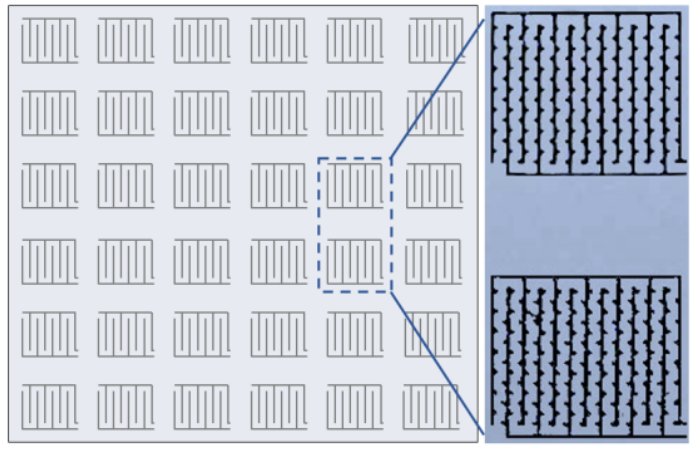

(a)

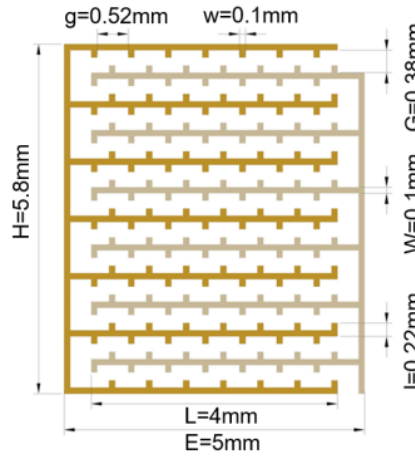

(b)

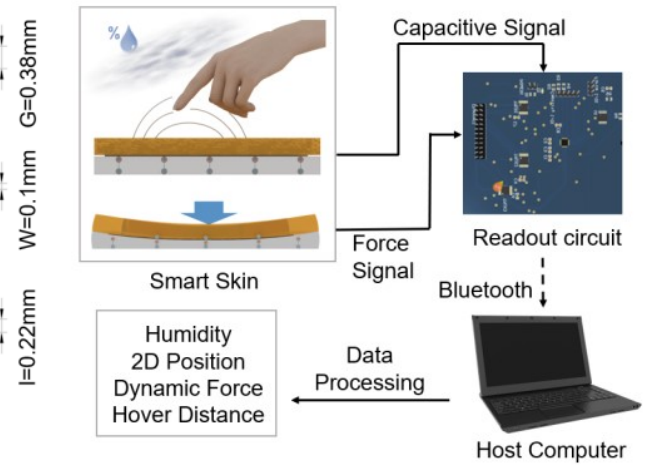

(c)

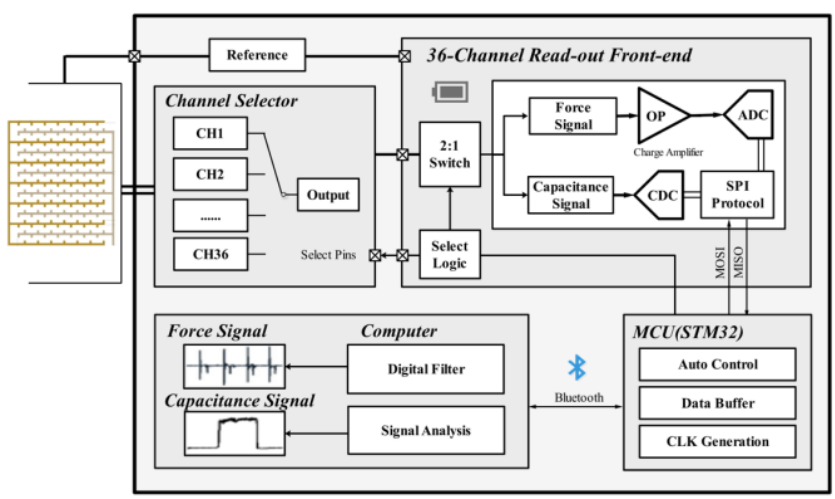

(d)

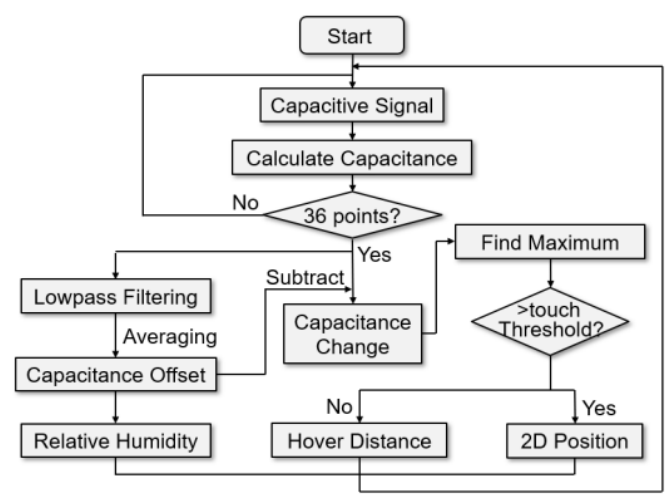

(e)

Fig. 2. (a) Conceptual top-view of the smart skin and a practical image of two interdigitated electrode pairs. (b) Geometric dimensions of the interdigitated electrodes. (c) Conceptual depiction of the device, circuitry and test configuration. (d) Block diagram of the readout circuit. (e) Flowchart of the algorithm for separating touch, proximity, and humidity.

are recognized by their capacitance changes when the device's surface electrode field is transformed by conductive objects, such as part of human bodies.

To capture force events, the piezoelectric-based technique is selected due to its passive mechanical-to-electric converting ability, which enables low system power consumption. Among conventional piezoelectric materials (including PVDF and its co-polymers [11-12], Zinc oxide [13-14], Barium titanite [15], etc.), PVDF film is utilized here to meet the high flexibility and force sensitivity requirement of electronic skins.

As shown in Fig. 2(a), a $6 \times 6$ electrode array is developed on a $100 \mu \mathrm{m}$-thickness polyimide film through a direct-writing technique. Each pair of electrodes has a thickness of $100 \mathrm{~nm}$ and occupies an area of $0.29 \mathrm{~cm}^{2}(0.5 \mathrm{~cm} \times 0.58 \mathrm{~cm})$. The width and interspacing of the interdigital fingers are $100 \mu \mathrm{m}$ and 380 $\mu \mathrm{m}$. In order to boost the mutual capacitance value, sub-fingers are introduced with finger lengths of $120 \mu \mathrm{m}$ and spacing of $520 \mu \mathrm{m}$. The geometric dimensions of an electrode pair are depicted in Fig. 2(b). A 50- $\mu$ m-thick PVDF film is finally laminated on the copper electrodes.

\section{B. System Construction}

The system's construction is conceptually demonstrated in Fig. 2(c). In force or hover touch experiments, both capacitive and force information are obtained by the readout circuit and uploaded to an upper computer. through subsequent algorithms, the signals are further separated and processed to be the force distribution, 2D position, hover distance, and environmental humidity.

Block diagram of the readout circuitry is shown in Fig. 2(d). The capacitance and force signals, which are both collected from the interdigital electrodes, are read in serial mode by a time-sharing circuit; the capacitive information is processed by capacitance-to-digital converters (CDCs), and force signals are transformed into voltage amplitudes by charge amplifiers and analogue-to-digital converters (ADCs). After that, the transformed digital messages are conveyed to the microprocessor unit through an $\mathrm{I} 2 \mathrm{C}$ protocol and transmitted to the host computer via wireless Bluetooth techniques.

To separate touch, proximity, and humidity information from the collected capacitive signals, a filtering algorithm is proposed (in Fig. 2(e)), which is based on the fact that the environmental humidity varies much more slowly than the hover and touch signals. The low-frequency component of mutual capacitance is utilized to calculate the relative humidity through an averaging process, and to serve as the baseline for contact and hover touch detection; the two situations are further determined by comparing the maximum capacitance change with a touch event threshold (discussed in section III).

\section{RESULTS AND DISSCUSSION}

This section will demonstrate the experimental results of the proposed sensing device and the whole system, together with discussions of the output performances. 


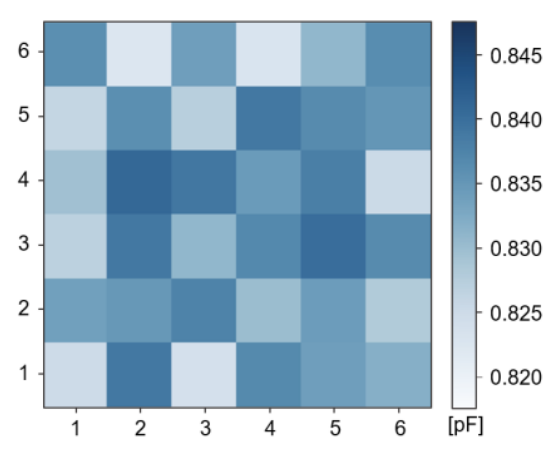

(a)

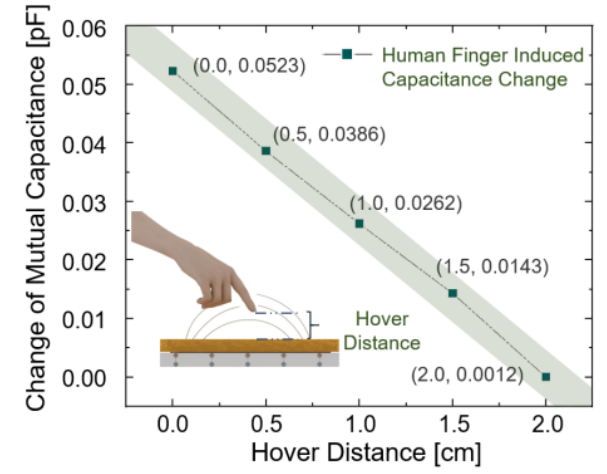

(b)

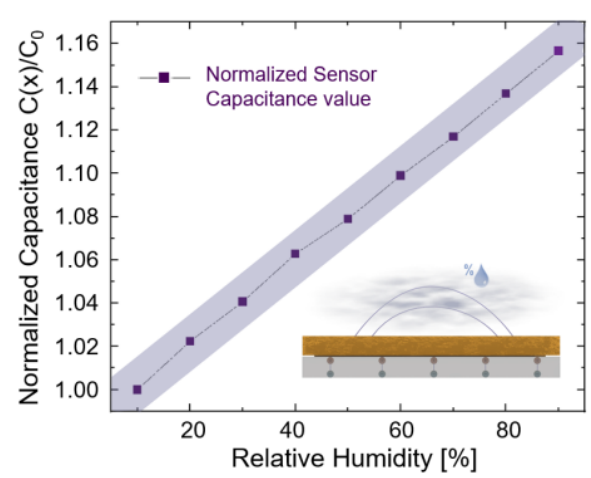

(c)

Fig. 3. (a) Mutual capacitance offsets of the device. (b) Human finger induced mutual capacitance changes under different hover distances. (c) Average mutual capacitance values of interdigitated electrodes under different relative humidity values.

Measurement of the device's mutual capacitances and dynamic forces are performed by a lock-in amplifier (MFLI, Zurich Instruments, Switzerland) and a push-pull dynamometer (HANDPI, Zhejiang, China), respectively, under room temperature $\left(20^{\circ} \mathrm{C}\right)$, with relative humidity at $42 \%$.

The overall mutual capacitance offset is measured first because it is vital to the determination of touch and hover event. As Fig. 3(a) shows, the experimental results show an average capacitance of $0.832 \mathrm{pF}$ with good uniformity $( \pm 0.011$ $\mathrm{pF})$. The average sensitivity of capacitance detection is examined to be $5.5 \pm 0.1 \mathrm{fF}$. In touching tests (Fig. 3(b)), the mutual capacitance change in a specific unit shows its proportional relationship to the finger proximity in a range of $0-2 \mathrm{~cm}$; the human finger induced capacitance change in a contact touch event is around $52 \mathrm{fF}$, half of which is therefore set as the touch detection threshold (26 fF), according to the widely-used determination method of capacitive touch events [16]. Humidity sensing is based on the increase of device's

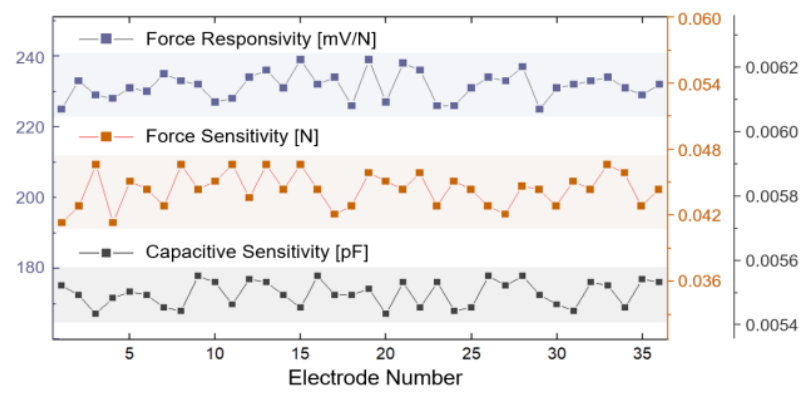

Fig. 4. Experimental performance of force and capacitive sensing.

TABLE I. SUMMARY OF SYSTEM PERFORMANCES

\begin{tabular}{|c|c|}
\hline \multicolumn{2}{|c|}{ System Parameters } \\
\hline Force Sensitivity & $0.051 \mathrm{~N}$ \\
\hline Force Responsivity & $2.47 \mathrm{~V} / \mathrm{N}$ \\
\hline Capacitive Sensitivity & $8.7 \mathrm{fF}$ \\
\hline Data Rate & $460,800 \mathrm{bps}$ \\
\hline ADC Resolution & $16 \mathrm{Bit}$ \\
\hline Capacitive Sampling Rate (36 points) & $80 \mathrm{~Hz}$ \\
\hline Force Sampling Rate (36 points) & $200 \mathrm{~Hz}$ \\
\hline
\end{tabular}

mutual capacitance in moisture sorption states, of which theoretical calculation has been demonstrated in [17]. Fig. 3(c) shows the humidity testing results, in which the device achieves an average responsivity at $0.20 \% / \mathrm{RH} \%$ in a relative humidity range of $10 \%-90 \% \mathrm{RH}$.

For dynamic force sensing, the total charges $(Q)$ collected by each electrode pairs are calculated by:

$$
Q=0.5 \times 0.31 \times d_{33} \times F
$$

where $F$ refers to the amplitude of external force and $d_{33}$ refers to the piezoelectric coefficient of the PVDF film. The preceding coefficients $(0.5$ and 0.31$)$ represents that one-third of the charges on a single side are collected. In experiments, the device's force sensing performances indicate an overall responsivity at $231.25 \pm 18 \mathrm{mV} / \mathrm{N}$ and sensitivity at $0.045 \pm$ $0.006 \mathrm{~N}$ for the 36-sensor array (shown in Fig. 4). The force sensing performance is lower than that found in [18-19] because the single-electrode layer collects only one side of the generated charges, and the interdigital electrodes occupy just one-third of the sensing area; however, as we talked above, this design offers more sensing functions.

System performances are examined with the configuration illustrated earlier in Fig. 2(c). The results, as summarized in TABLE I, indicate average force and capacitive sensitivity at $0.051 \mathrm{~N}$ and $8.7 \mathrm{fF}$, respectively. The force responsivity achieved $2.47 \mathrm{~V} / \mathrm{N}$, which is higher than that of the device because of the readout circuits' amplification.

\section{CONCLUSION}

In this article, we present a piezoelectric- and capacitivebased flexible smart skin for multidimensional sensing. With a post-processing system and comprehensive algorithm, the smart skin shows its capabilities of simultaneous detecting and differentiating various environmental stimuli. Our experimental results demonstrate that this technique successfully achieves desired attributes (e.g., flexibility, multiple-function, large area, and low cost) for humanoid robots' applications, hence potentially reinforcing their use in various scenarios, such as factory assembly line work, surgery operation, and human-machine interactions. 


\section{REFERENCES}

[1] B. Robins, K. Dautenhahn, R. Te Boekhorst, and A. Billard, "Robotic assistants in therapy and education of children with autism: can a small humanoid robot help encourage social interaction skills?," Universal Access in the Information Society, vol. 4, no. 2, pp. 105-120, 2005.

[2] K. Dautenhahn, "Robots as social actors: Aurora and the case of autism," in Proc. CT99, The Third International Cognitive Technology Conference, August, San Francisco, 1999, vol. 359, p. 374.

[3] J. Wainer, K. Dautenhahn, B. Robins, and F. Amirabdollahian, "Collaborating with Kaspar: using an autonomous humanoid robot to foster cooperative dyadic play among children with autism," in 2010 10th IEEE-RAS International Conference on Humanoid Robots, 2010: IEEE, pp. 631-638.

[4] L. P. E. Toh, A. Causo, P.-W. Tzuo, I.-M. Chen, and S. H. Yeo, "A review on the use of robots in education and young children," Journal of Educational Technology \& Society, vol. 19, no. 2, pp. 148-163, 2016.

[5] T. Shibata and K. Wada, "Robot therapy: a new approach for mental healthcare of the elderly-a mini-review," Gerontology, vol. 57, no. 4, pp. 378-386, 2011.

[6] P.-C. Yang, K. Sasaki, K. Suzuki, K. Kase, S. Sugano, and T. Ogata, "Repeatable folding task by humanoid robot worker using deep learning," IEEE Robotics and Automation Letters, vol. 2, no. 2, pp. 397-403, 2016.

[7] B. C.-K. Tee et al., "A skin-inspired organic digital mechanoreceptor," Science, vol. 350, no. 6258, pp. 313-316, 2015.

[8] H.-H. Chou et al., "A chameleon-inspired stretchable electronic skin with interactive colour changing controlled by tactile sensing," Nature communications, vol. 6, no. 1, pp. 1-10, 2015.

[9] M. Shi et al., "Self-powered analogue smart skin," ACS nano, vol. 10, no. 4, pp. 4083-4091, 2016.

[10] S. Harada, K. Kanao, Y. Yamamoto, T. Arie, S. Akita, and K. Takei, "Fully printed flexible fingerprint-like three-axis tactile and slip force and temperature sensors for artificial skin," ACS nano, vol. 8, no. 12, pp. 12851-12857, 2014.
[11] B. Mahanty, K. Maity, S. Sarkar, and D. Mandal, "Human skin interactive self-powered piezoelectric e-skin based on PVDF/MWCNT electrospun nanofibers for non-invasive health care monitoring," Materials Today: Proceedings, vol. 21, pp. 1964-1968, 2020.

[12] S. Gupta, L. Lorenzelli, and R. Dahiya, "Multifunctional flexible PVDF-TrFE/BaTiO 3 based tactile sensor for touch and temperature monitoring," in 2017 IEEE SENSORS, 2017: IEEE, pp. 1-3.

[13] W. Deng et al., "A flexible field-limited ordered $\mathrm{ZnO}$ nanorod-based self-powered tactile sensor array for electronic skin," Nanoscale, vol. 8, no. 36, pp. 16302-16306, 2016.

[14] M.-S. Suen, Y.-C. Lin, and R. Chen, "A flexible multifunctional tactile sensor using interlocked $\mathrm{ZnO}$ nanorod arrays for artificial electronic skin," Procedia Engineering, vol. 168, pp. 1044-1047, 2016.

[15] Y. Yang et al., "Flexible piezoelectric pressure sensor based on polydopamine-modified BaTiO3/PVDF composite film for human motion monitoring," Sensors and Actuators A: Physical, vol. 301, p. 111789, 2020.

[16] C.-L. Lee, S.-F. Tai, J.-M. Liu, and C.-F. Hsieh, "Method for determining position of touch event on touch panel by dynamically adjusting threshold and related computer readable medium," ed: Google Patents, 2017.

[17] H. Shibata, M. Ito, M. Asakursa, and K. Watanabe, "A digital hygrometer using a polyimide film relative humidity sensor," IEEE Transactions on Instrumentation and Measurement, vol. 45, no. 2, pp. 564-569, 1996.

[18] S. Jeon, S.-C. Lim, T. Q. Trung, M. Jung, and N.-E. Lee, "Flexible multimodal sensors for electronic skin: principle, materials, device, array architecture, and data acquisition method," Proceedings of the IEEE, vol. 107, no. 10, pp. 2065-2083, 2019.

[19] Y. Gu et al., "Mini review on flexible and wearable electronics for monitoring human health information," Nanoscale research letters, vol. 14, no. 1, pp. 1-15, 2019. 